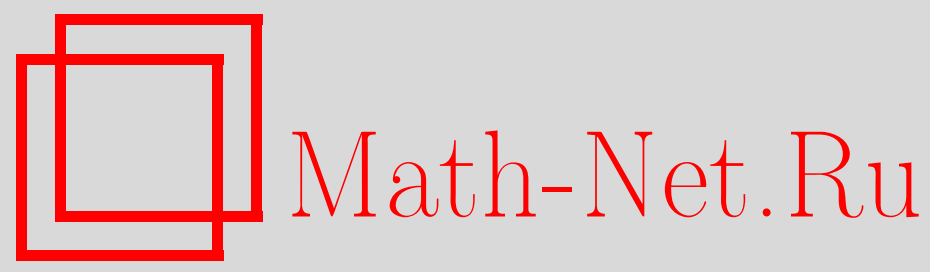

Д. Реповш, М. Б. Скопенков, М. Ценцель, Элементарное доказательство теоремы о 12 целых точках, Матем. заметки, 2005, том 77, выпуск 1, 117-120

DOI: https://doi.org/10.4213/mzm2474

Использование Общероссийского математического портала Math-Net.Ru подразумевает, что вы прочитали и согласны с пользовательским соглашением http://www . mathnet.ru/rus/agreement

Параметры загрузки:

IP : 18.207 .199 .55

26 апреля 2023 г., 12:24:01

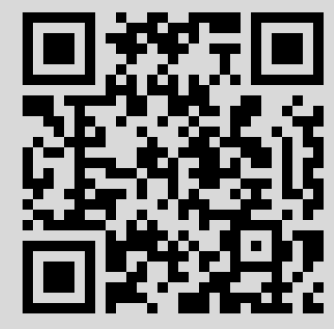




\title{
ЭЛЕМЕНТАРНОЕ ДОКАЗАТЕЛЬСТВО ТЕОРЕМЫ О 12 ЦЕЛЫХ ТОЧКАХ
}

\author{
Д. Реповш, М. Скопенков, М. Ценцель
}

\begin{abstract}
В данной заметке приводится элементарное доказательство следующей теоремы (теорема о 12 целых точках). Пусть $M$ - выпуклый многоугольник с вершинами в целых точках, содержащий внутри себя ровно одну целую точку. Обозначим через $m$ количество целых точек на границе многоугольника $M$, а через $m^{*}$ - на границе двойственного к нему многоугольника. Тогда $m+m^{*}=12$.
\end{abstract}

Библиография: 3 названия.

Теорема о 12 целых точках - красивое и простое по формулировке утверждение, у которого до последнего времени не было простого доказательства. Данная заметка посвящена ее элементарному доказательству. Для формулировки нам понадобится

ОПРЕДЕЛЕНИЕ ДВОЙСТВЕНнОГО МНОГОУГОЛЬНИКА. Пусть на Плоскости фиксирована декартова система координат. Рассмотрим выпуклый многоугольник $M=$ $A_{1} A_{2} \ldots A_{n}$ с вершинами в целых точках, содержащий внутри себя ровно одну целую точку $O$ (см. рис. 1 ). Отложим от точки $O$ векторы сторон многоугольника $\overrightarrow{A_{1} A_{2}}, \overrightarrow{A_{2} A_{3}}, \ldots, \overrightarrow{A_{n} A_{1}}$ и на каждом из них отметим ближайшую к $O$ целую точку (отличную от $O$ ). Соединяя последовательно отмеченные точки, получим многоугольник $M^{*}$, двойственный исходному. Обозначим количество целых точек на гранищах многоугольников $M$ и $M^{*}$ через $m$ и $m^{*}$ соответственно.
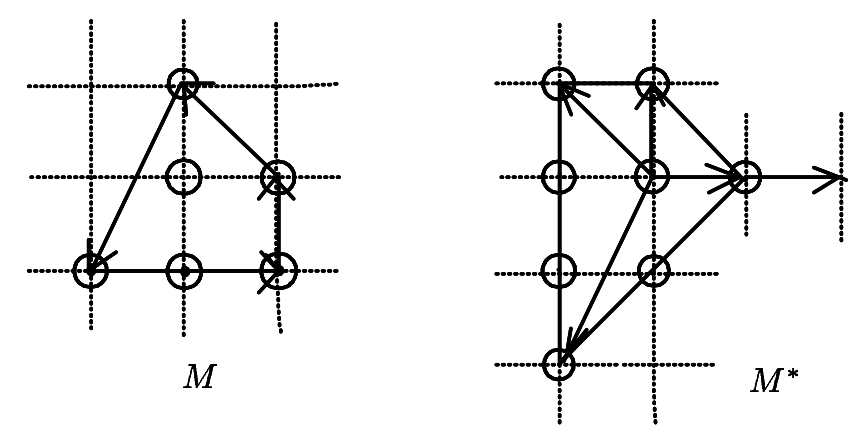

Рис. 1

Работа первого и третьего авторов выполнена при частичной поддержке Министерства образования, науки и спорта Республики Словения, грант № 101-509. Работа второго автора выполнена при частичной поддержке Российского фонда фундаментальных исследований, гранты № 03-0110825 и № 02-01-00014. 
ТЕОРЕМА О 12 ЦЕЛЫХ ТОЧКАХ. Пусть $M-$ выпуклый многоугольник $с$ вериинами в иелых точках, содержащий внутри ровно одну челую точку. Тогда

$$
m+m^{*}=12 \text {. }
$$

Эта теорема была сформулирована в книге [1] с некоторыми указаниями к доказательству, использующему технику торических многообразий (см. также [2]). В интересной статье [3], целиком посвященной этой теореме, обсуждаются четыре различных доказательства. Три из них довольно объемны и основаны соответственно на сведении к перебору 16 вариантов, теории модулярных форм и теории торических многообразий. Идея четвертого очень близка к идее данной заметки. (Для него в указанной статье приводится только план, прямая реализация которого также довольно громоздка.)

Приведенное ниже элементарное доказательство аналогично одному из доказательств формулы Пика. Мы сводим теорему о 12 целых точках к частному случаю, когда $M-$ параллелограмм и $m=4$. Начнем именно с этого случая.

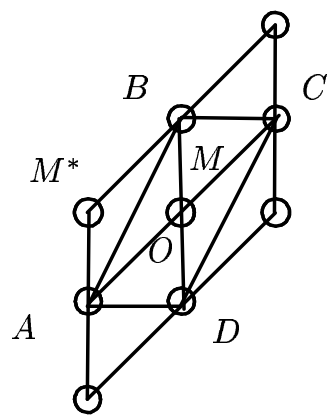

Рис. 2

(1) Если $M=A B C D$ - параллелограмм без иельх точек на сторонах, то $m+m^{*}=12$ (см. рис. 2 ).

Действительно, в этом случае $O=A C \cap B D$. Это следует из того, что точка, симметричная точке $O$ относительно $A C \cap B D$, целая и лежит внутри $A B C D$, а поэтому совпадает с $O$. Легко видеть, что $M^{*}$ - параллелограмм, стороны которого получаются из диагоналей $A C$ и $B D$ параллельньми переносами на векторы $\pm \overrightarrow{O B}$ и $\pm \overrightarrow{O A}$ соответственно. Так как на этих диагоналях лежит единственная целая точка $O$, то на каждой сторонепараллелограмма $M^{*}$ лежит по одной целой точке, откуда $m+m^{*}=4+8=12$.

Пусть теперь $M=A_{1} \ldots A_{n}$. Будем считать все целые точки на сторонах многоугольника $M$ вершинами (возможно, с углом $180^{\circ}$ ). На определении $M^{*}$ это не отразится. Предположим, что некоторьй треугольник $A_{i-1} A_{i} A_{i+1} n p o c m$, т.е. не содержит целых точек, отличных от вершин (ни внутри, ни на сторонах). Элементарной операиией $A_{1} \ldots A_{i-1} A_{i} A_{i+1} \ldots A_{n} \rightarrow A_{1} \ldots A_{i-1} A_{i+1} \ldots A_{n}$ назовем операцию удаления треугольника $A_{i-1} A_{i} A_{i+1}$ из $M$ или обратную к ней. Наш метод сведения основан на следующем утверждении.

(2) При әлементарной операции величина $m+m^{*}$ сохраняется.

Нам достаточно доказать, что, например, удаление простого треугольника $A_{1} A_{2} A_{3}$ из $M$ приводит к добавлению простого треугольника $A_{12} A_{13} A_{23} \mathrm{~K} M^{*}$ (см. рис. 3 ). 

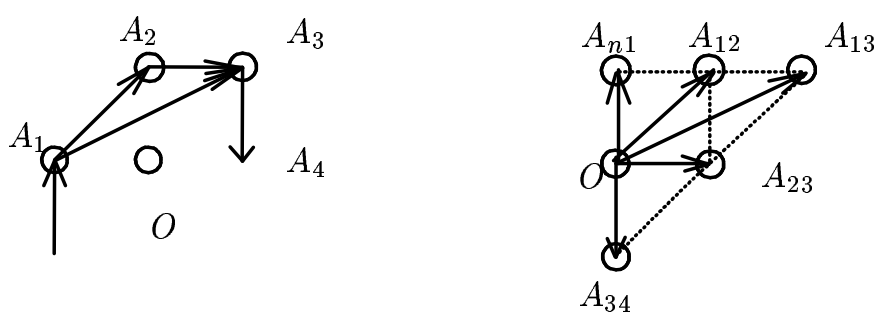

Рис. 3

Здесь через $A_{k l}$ обозначена точка такая, что $\overrightarrow{O A_{k l}}=\overrightarrow{A_{k} A_{l}}$. В частности, если $l=k+1$, то $A_{k l}$ - вершина многоугольника $M^{*}$. Удалим $A_{1} A_{2} A_{3}$. Тогда у многоугольника $M^{*}$ исчезнут вершины $A_{12}$ и $A_{23}$, зато добавится новая вершина $A_{13}$. Ее еще нужно соединить отрезками с $A_{n 1}$ и $A_{34}$. Покажем, что точки $A_{12}$ и $A_{23}$ лежат на этих отрезках. В самом деле, так как $O$ - единственная целая точка внутри $M$, то треугольники $A_{1} O A_{3}$, $A_{2} O A_{3}, A_{4} O A_{3}$ простые. Из формулы Пика следует, что их площади равны $1 / 2$. Поскольку они имеют общее основание $O A_{3}$, то проекции векторов $\overrightarrow{A_{1} A_{3}}, \overrightarrow{A_{1} A_{3}}$ и $\overrightarrow{A_{1} A_{3}}$ на перпендикуляр к $O A_{3}$ равны. Отсюда следует, что точки $A_{13}, A_{23}$ и $A_{34}$ лежат на одной прямой, а так как $M$ вьпуклый, то $A_{23}$ лежит между двумя остальными. Аналогично доказьвается, что $A_{12}$ принадлежит отрезку $A_{n 1} A_{13}$. Значит, преобразование $M^{*}$ сводится к добавлению треугольника $A_{12} A_{13} A_{23}$. Заметим, что треугольник $O A_{12} A_{13}$ получается из простого треугольника $A_{1} A_{2} A_{3}$ параллельным переносом, а $O A_{23} A_{13}-$ центральной симметрией. Поэтому треугольник $A_{12} A_{13} A_{23}$ простой, что и требовалось.

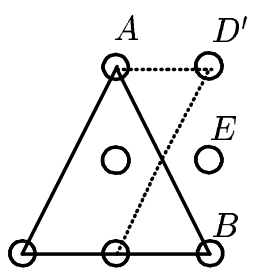

B $\quad C$

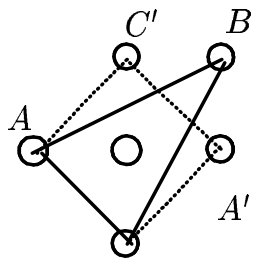

C

Рис. 4

Для доказательства теоремы остается заметить следующее.

(3) Из любого многоугольника $M$ серией әлементарных операций можно получить параллелограмм без челых точек на сторонах.

Действительно, предположим вначале, что у $M$ есть диагональ, не проходящая через $O$. Разрежем $M$ вдоль этой диагонали и рассмотрим ту из полученных частей, которая не содержит $O$. Эта часть обязательно содержит простой треугольник вида $A_{i-1} A_{i} A_{i+1}$. Поэтому элементарной операцией можно уменьшить число $m$. Будем действовать так, пока это возможно. Очевидно, есть только три случая, когда требуемой диагонали не найдется:

A) $m=4, M=A B C D, O=A C \cap B D$; так как отрезки $O A, O B, O C$ и $O D$ не содержат целых точек, то $O A=O C$ и $O B=O D$, т.е. $A B C D$ - искомый параллелограмм; 
В) $m=4, M=A B C D$, один из углов, скажем $B C D$, развернутьй; в этом случае обозначим через $D^{\prime}$ точку, симметричную точке $D$ относительно $O$, через $E-$ середину $D^{\prime} B$; искомая серия элементарных операций имеет вид

$$
A B C D \rightarrow A E B C D \rightarrow A D^{\prime} E B C D \rightarrow A D^{\prime} E C D \rightarrow A D^{\prime} C D
$$

(см. рис. 4);

C) $m=3, M=A B C$; в этом случае обозначим через $A^{\prime}$ и $C^{\prime}$ точки, симметричные относительно $O$ вершинам $A$ и $C$ соответственно; тогда искомая серия имеет вид

$$
A B C \rightarrow A C^{\prime} B C \rightarrow A C^{\prime} B A^{\prime} C \rightarrow A C^{\prime} A^{\prime} C
$$

(см. рис. 4).

Теорема доказана.

Благодарности. Авторы благодарны В. В. Прасолову за постановку задачи.

\section{СПИСОК ЦИТИРОВАННОЙ ЛИТЕРАТУРЫ}

[1] Fulton W. Introduction to Toric Varieties. New Jersey: Princeton, 1993.

[2] Хованский А. Г. Многоугольники Ньютона, кривые на торических поверхностях и обращение теоремы Вейля // УМН. 1997. Т. 52. №6. С. 113-142.

[3] Poonen B., Rodriges-Villegas F. Lattice polygons and the number 12 // Amer. Math. Month. 2000. V. 107. № 3. P. 238-250.

(Д. Реповш, М. Ценцель) Университет Любляны, Словения 